\title{
Inhibition of DTYMK significantly restrains the growth of HCC and increases sensitivity to oxaliplatin
}

Fengze Sun (iD ${ }^{1,3}$, Yuanyuan Liu ${ }^{1,3}$, Tingting Gong ${ }^{2,3}$, Qiuzhong Pan ${ }^{1}$, Tong Xiang ${ }^{1}$, Jingjing Zhao ${ }^{1}$, Yan Tang ${ }^{1}$, Hao Chen ${ }^{1}$, Yulong Han ${ }^{1}$, Mengjia Song ${ }^{1}$, Yue Huang ${ }^{1}$, Han $\mathrm{Li}^{1}$, Yuanyuan Chen ${ }^{1}$, Chaopin Yang ${ }^{1}$, Jieying Yang ${ }^{1}$, Qijing Wang ${ }^{1}$, Yongqiang $\mathrm{Li}^{1}$, Jia $\mathrm{He}^{1}$, Desheng Weng ${ }^{1}$, Ruiqing Peng $\left(\mathbb{D}^{1 凶}\right.$ and Jianchuan Xia ${ }^{1 \mathrm{D}}{ }^{\mathrm{m}}$

(c) The Author(s) 2021

Most patients with hepatocellular carcinoma (HCC) are in the middle or advanced stage at the time of diagnosis, and the therapeutic effect is limited. Therefore, this study aimed to verify whether deoxythymidylate kinase (DTYMK) increased in HCC and was an effective therapeutic target in HCC. The findings revealed that the DTYMK level significantly increased and correlated with poor prognosis in HCC. However, nothing else is known, except that DTYMK could catalyze the phosphorylation of deoxythymidine monophosphate (dTMP) to form deoxythymidine diphosphate (dTDP). A number of experiments were performed to study the function of DTYMK in vitro and in vivo to resolve this knowledge gap. The knockdown of DTYMK was found to significantly inhibit the growth of HCC and increase the sensitivity to oxaliplatin, which is commonly used in HCC treatment. Moreover, DTYMK was found to competitively combine with miR-378a-3p to maintain the expression of MAPK activated protein kinase 2 (MAPKAPK2) and thus activate the phospho-heat shock protein 27 (phospho-HSP27)/nuclear factor NF-kappaB (NF-KB) axis, which mediated the drug resistance, proliferation of tumor cells, and infiltration of tumor-associated macrophages by inducing the expression of $\mathrm{C}-\mathrm{C}$ motif chemokine ligand 5 (CCL5). Thus, this study demonstrated a new mechanism and provided a new insight into the role of mRNA in not only encoding proteins to regulate the process of life but also regulating the expression of other genes and tumor microenvironment through the competing endogenous RNA (ceRNA) mechanism.

Cell Death and Disease (2021)12:1093; https://doi.org/10.1038/s41419-021-04375-3

\section{INTRODUCTION}

The 5 -year relative survival rate of liver cancer is only $20 \%$, and the incidence rate is rising annually [1]. Hepatocellular carcinoma (HCC) is the most common type of all liver cancers (comprising $80 \%$ of cases). Infection with hepatitis B virus (HBV) and hepatitis $C$ virus, alcoholic liver disease, and most probably nonalcoholic fatty liver disease are major risk factors for HCC. Among them, chronic HBV infection accounts for $\sim 50 \%$ of all cases of HCC [2]. Most patients with HCC are in the middle or advanced stage at the time of diagnosis, with a high degree of malignancy, easy recurrence, and poor prognosis, which seriously threatens their health and life [3, 4]. The treatment of HCC often requires multidisciplinary knowledge, including surgery, hepatology, interventional radiology, oncology, and so forth $[5,6]$. Orthotopic liver transplantation or surgical resection is considered the most effective treatment; alternative nonsurgical treatments include microwave coagulation therapy, percutaneous acetic acid injection, laser interstitial thermal ablation therapy, radiofrequency ablation, and cryoablation therapy [7, 8]. However, the long-term therapeutic effect of HCC remains unsatisfactory, especially in patients with advanced unresectable disease. Oxaliplatin is one of the most commonly used chemotherapeutic drugs in transcatheter arterial chemoembolization, hepatic arterial infusion, and systemic administration of HCC [9-11], although oxaliplatin resistance is also an important reason for poor therapeutic effect and recurrence of $\mathrm{HCC}[12,13]$. Hence, finding effective markers and targets of HCC and increasing the sensitivity to drugs are critical to improving the prognosis of patients with HCC.

DNA synthesis is an essential prerequisite for cell replication, especially in tumor cells. Therapeutic agents that target deoxyribonucleoside triphosphate synthesis and metabolism are commonly used in the clinical treatment of several cancer types [14]. The deoxythymidine-5'-monophosphate (dTMP) is synthesized from the methylation of deoxyuridine-5'-monophosphat by thymidylate synthase in the de novo pathway [15]. In the salvage pathway, dTMP is produced from the phosphorylation of thymidine by thymidine kinase. Deoxythymidylate kinase (DTYMK) can catalyze the phosphorylation of dTMP to form dTDP. Besides, it is the first merged step of both salvage and de novo pathways in the production of dTTP, which is an important material for DNA synthesis [16, 17]. Previous studies reported that the knockdown of DTYMK inhibited this pathway, leading to a decrease in the product $\mathrm{dTDP}$ and the accumulation of the substrate dTMP [18]. However, nothing else is known, except that

\footnotetext{
${ }^{1}$ Department of Biotherapy, State Key Laboratory of Oncology in Southern China, Collaborative Innovation Center for Cancer Medicine, Sun Yat-sen University Cancer Center, Guangzhou, Guangdong, China. ${ }^{2}$ Department of Ultrasound, The Fifth Affiliated Hospital of Sun Yat-Sen University, Zhuhai, Guangdong, China. ${ }^{3}$ These authors contributed equally: Fengze Sun, Yuanyuan Liu, Tingting Gong. ${ }^{凶}$ email: pengrq@sysucc.org.cn; xiajch@mail.sysu.edu.cn Edited by Professor Stephen Tait
} 
A

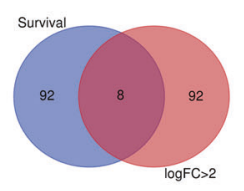

B

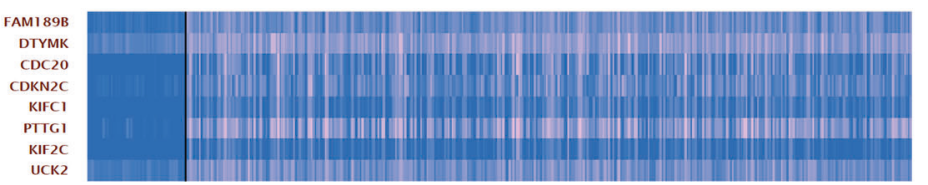

C

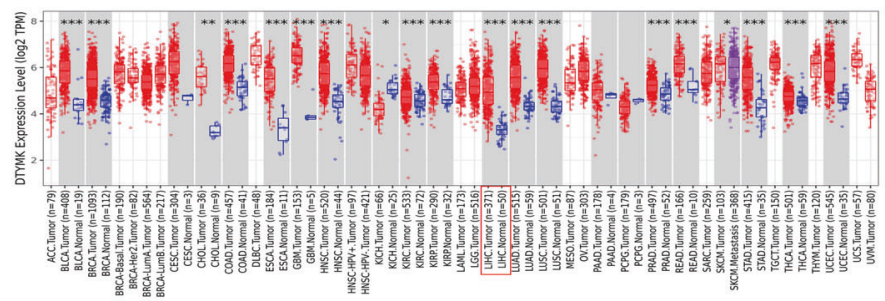

E

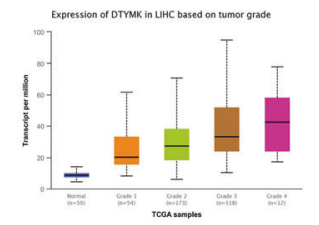

F

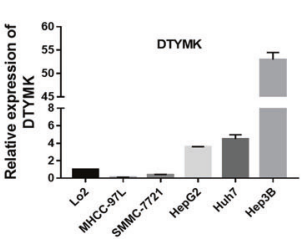

G

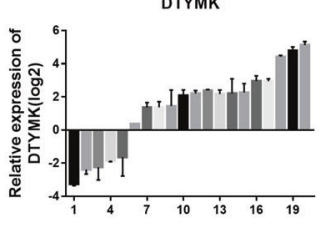

D

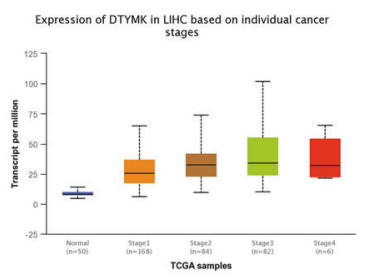

$\mathrm{H}$

Fig. 1 Increased expression of DTYMK in HCC. A Gene analysis of patients with HCC from TCGA. B Eight genes were upregulated and had the most significant survival differences (C) DTYMK expression increased in multiple cancer types, especially in HCC. D Expression of DTYMK was related to cancer stages. E Expression of DTYMK was related to cancer grades. F Increased RNA level of DTYMK in HepG2, Huh7, and Hep3B HCC cell lines. G Increased RNA level of DTYMK in most patients with HCC. H Increased protein level of DTYMK in most patients with HCC.

DTYMK could catalyze the phosphorylation of dTMP to form dTDP. Few studies have reported on the role of DTYMK, particularly in cancer occurrence and progression. According to the results of The Cancer Genome Atlas (TCGA), the expression of DTYMK increased and associated with a poor prognosis in several cancers. Thus, this study aimed to verify whether DTYMK expression increased in HCC and was an effective therapeutic target in HCC.

\section{RESULTS}

\section{Increased expression of DTYMK in HCC}

After analyzing HCC data from the TCGA, the top 100 mRNAs were upregulated most significantly (fold change $>2$ ), and the top 100 genes with the most significant survival differences were intersected to obtain the critical genes in HCC genesis and development (Fig. 1A). Eight genes fitted the inclusion criteria: FAM189B, DTYMK, CDC20, CDKN2C, KIFC1, PTTG1, KIF2C, and UCK2 (Fig. 1B). Among these genes, this study focused on DTYMK. No in-depth studies on DTYMK have been reported to date. DTYMK was found to be highly expressed in Pan-Cancer Atlas, especially in HCC (Fig. 1C). Besides, the expression of DTYMK was related to tumor stages and grades in HCC according to the results of TCGA (Figs. 1D and E). Whether DTYMK expression increased in HCC was verified by examining $20 \mathrm{HCC}$ tissues and paired adjacent nontumor tissues and HCC cell lines using quantitative polymerase chain reaction (qPCR) and Western blot assays. DTYMK was found to increase in HepG2, Hun7, and Hep3B HCC cell lines compared with LO2 hepatic epithelial cells and in most HCC tissues (Fig. 1F-H).

\section{Decreased expression of DTYMK inhibited HCC growth and increased sensitivity to oxaliplatin}

Figure 2A shows how DTYMK was involved in DNA synthesis. After the knockdown of DTYMK, the proliferation of Hep3B and Huh7 HCC cells was significantly inhibited (Fig. $2 \mathrm{~B}$ and $\mathrm{C}$ ), while the growth of the tumor cells was partially restored after the addition of dTDP, which was the product of DTYMK. The cell cycle experiment showed that the G0/G1 phase was prolonged and the
$S$ phase was shortened after DTYMK knockdown in Huh7 and Hep3B cell lines (Fig. 2D and E). In addition, the levels of cell cycle proteins CDK2, CDK4, Cyclin A2, and Cyclin D1, which were involved in the G0/G1 and $S$ phases, were significantly reduced after DTYMK knockdown (Fig. 2F). Besides, Huh7 and Hep3B cells became more sensitive to oxaliplatin, which was a commonly used chemotherapeutic drug for HCC, after DTYMK knockdown (Fig. 2G and $\mathrm{H}$ ). The cleavage of Poly ADP-Ribose Polymerase (PARP) during apoptosis has been reported to facilitate cellular disassembly and ensure the completion and irreversibility of the process [19]. Bcl-2/Bax is widely reported as a rheostat that regulates cell death [20]. In this study, cleaved-PARP and Bax increased and $\mathrm{Bcl}-2$ decreased in the sh-DTYMK group than in the other groups (Fig. 2l). The sensitivity to sorafenib also increased after DTYMK knockdown (Supplementary Fig. 1).

\section{DTYMK acted as ceRNA to affect the function of MAPKAPK2}

To find out how DTYMK affected the growth of HCC and sensitivity to oxaliplatin, data from TCGA were analyzed using starBASE v2.0 [21]. The results revealed that DTYMK could act as an endogenous competitive RNA (ceRNA) to affect the function of several genes, including MAPKAPK2, AKT1, CART, and NRF1, by binding to microRNA-378a-3p (miR-378a-3p). It was reported that miR-378a$3 p$ could play important roles in cancer development [22]. The results of TCGA, GSE74618, and GSE153089 showed that miR378a-3p significantly decreased in HCC than in normal tissues $[23,24]$ (Fig. 3A). Similarly, RNA sequencing (RNA-seq) of six paired $\mathrm{HCC}$ and normal liver tissues and QPCR results of 20 paired HCC and normal liver tissues from Sun Yat-sen University Cancer Center showed that miR-378a-3p significantly decreased in HCC (Fig. 3B and C). MAPKAPK2, AKT1, CART, and NRF1 could be inhibited by miR-378a-3p, and their expression decreased after DTYMK knockdown (Fig. $3 \mathrm{D}$ and E). According to the clustering analysis of RNA-seq, MAPKAPK2 showed quite similar expression trends with DTYMK ( $r=0.585, p=0.046)$ (Fig. 3F and G). Besides, miR378a-3p with an opposite expression trend of DTYMK $(r=-0.377$, $p=0.228)$ and MAPKAPK2 $(r=-0.546, p=0.066)$, although the difference was not significant due to the insufficient number of samples (Fig. $3 \mathrm{H}$ and I). Highly relevant expression of DTYMK and 

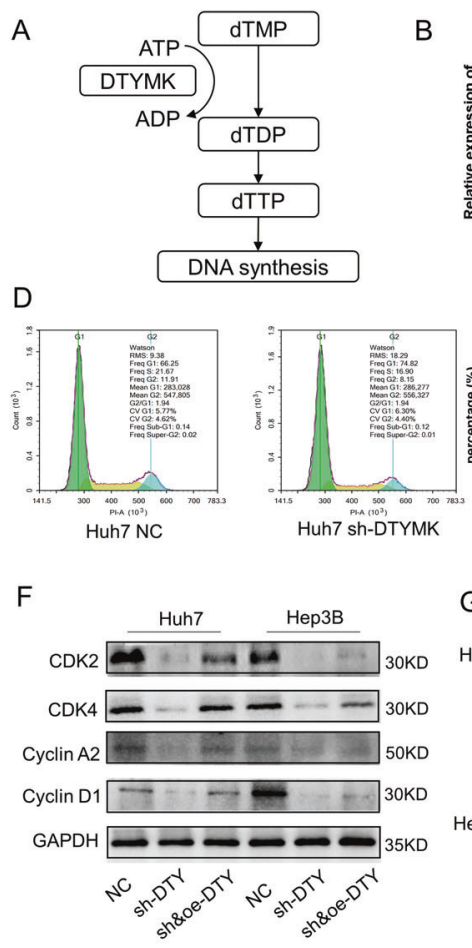

$\mathrm{H}$

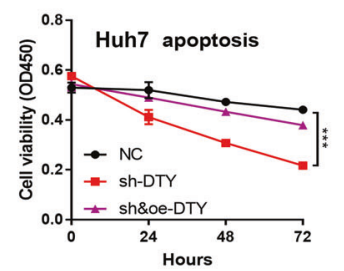

B

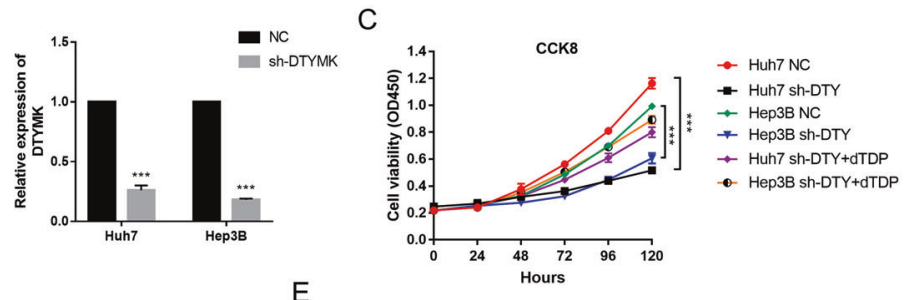

E
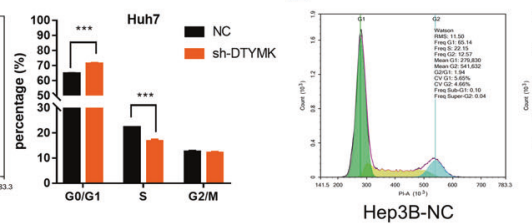

Hep3B-NC
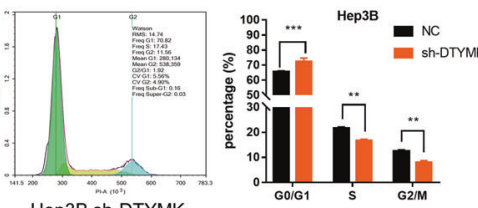

Hep3B sh-DTYMK
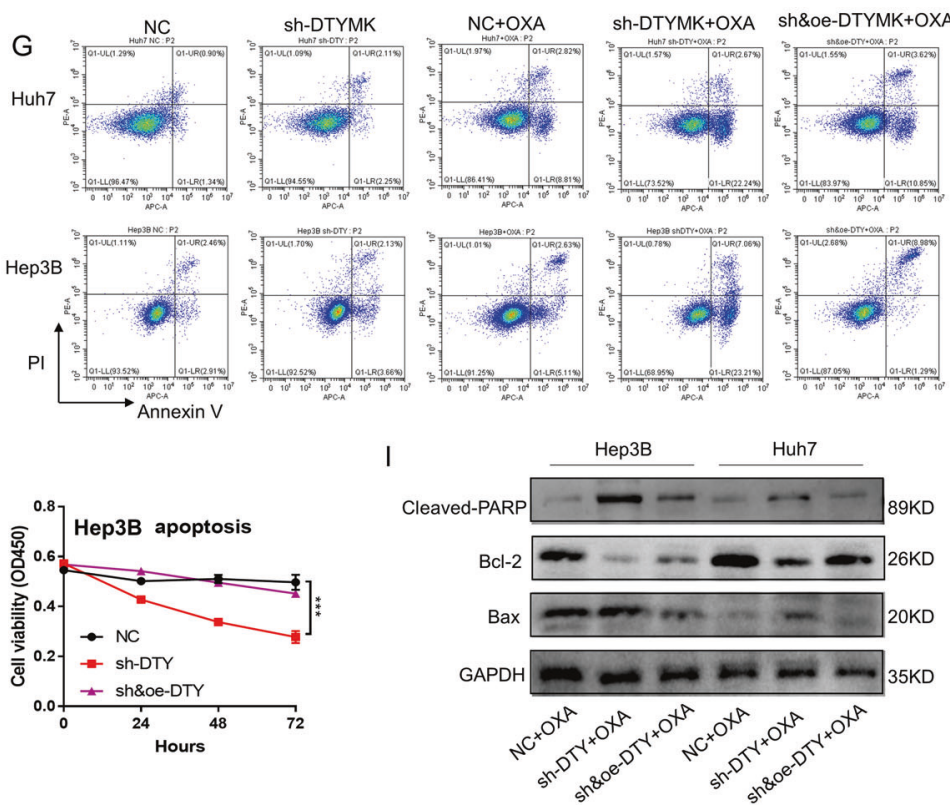

Fig. 2 Decreased expression of DTYMK inhibited proliferation and increased sensitivity to oxaliplatin in HCC cells. A DTYMK catalyzed the phosphorylation of dTMP to form dTDP. B and C Decreased DTYMK significantly inhibited the proliferation of Huh7 and Hep3B cells, while the supplement of dTDP partly rescued the proliferation ability. D and E Decreased expression of DTYMK could lead to cell cycle arrest and reduce the proportion of cells entering the G1 phase. F Decreased expression of CDK2, CDK4, Cyclin A2, and Cyclin D1 after DTYMK knockdown. G and $\mathbf{H}$ Increased sensitivity to oxaliplatin after DTYMK knockdown. I Reduced Bcl-2 and increased cleaved-PARP and Bax in the sh-DTYMK group than in the other groups. DTY, DTYMK; OXA, Oxaliplatin. ${ }^{*} p<0.05 ;{ }^{* *} p<0.01 ;{ }^{* * *} p<0.001$.

MAPKAPK2 was also found in HCC $(r=0.229, p<0.001)$, kidney chromophobe $(\mathrm{KICH}, r=0.468, p<0.001)$, thyroid carcinoma (THCA, $r=0.281, p<0.001)$, pancreatic adenocarcinoma $(r=$ $0.333, p<0.001)$, brain lower-grade glioma $(r=0.476, p<0.001)$, and uveal melanoma $(r=0.318, p=0.004)$ (Fig. 3J). MAPKAPK2 expression increased in 15 of $20 \mathrm{HCC}$ tissues (Fig. 3K), which was highly similar to the DTYMK expression $(r=0.630, p<0.01)$ (Fig. $3 \mathrm{~L}$ ). Similar to the RNA-seq, the results of qPCR from the 20 clinical samples showed miR-378a-3p had an opposite expression trend of DTYMK $(r=-0.839, p<0.001)$ and MAPKAPK2 $(r=$ $-0.565, p=0.01$ ) (Fig. $3 \mathrm{M}$ and N). In addition, the RNA of DTYMK and MAPKAPK2 both located mainly in the cytoplasm, which was considered as a necessary condition for working through ceRNA (Fig. 30). MAPKAPK2 could be significantly inhibited after DTYMK knockdown, and thereby inhibit the phosphorylation of heat shock protein 27 (HSP27), which could promote nuclear factor NF-kappaB (NF-kB) in the nucleus (Fig. 3P). Potential binding sites between DTYMK, MAPKAPK2, and miR-378a-3p are shown in Fig. 3Q. Fluorescence intensity was evaluated using dual-luciferase reporter assay after co-transfection with miR-378a-3p mimics to investigate whether miR-378a-3p could inhibit DTYMK and
MAPKAPK2 (Fig. 3R). The Western blot analysis showed that miR-378a-3p could significantly inhibit DTYMK/MAPKAPK2/pHSP27 and prevent NF-KB in the nucleus (Fig. 3S).

\section{Tumor formation rate and growth speed were significantly inhibited after DTYMK knockdown in vivo}

After DTYMK knockdown, a stronger inhibitory effect on tumor formation rate (tumors formatted in only 3 in 10 mice in the shDTYMK group) and tumor growth speed $(p=0.039)$ was observed in transplanted carcinoma in nude mice (Fig. 4A-C). The tumor sizes in the negative control group decreased partially after oxaliplatin injection, while no tumors were formatted in the shDTYMK group (Fig. 4D). A schematic diagram of the tumor xenotransplantation model is shown in Fig. 4E. The results of immunohistochemistry showed that the expression of DTYMK and MAPKAPK2 reduced in the sh-DTYMK group compared with the negative control group (Fig. 4F). Besides, more $\mathrm{CD} 163^{+} \mathrm{M} 2$ tumorassociated macrophages (TAMs) were found to infiltrate into the tumor and adjacent stroma. This might be due to the higher expression of $\mathrm{C}-\mathrm{C}$ motif chemokine ligand 5 (CCL5), which was a powerful chemokine to recruit monocytes and could be regulated 

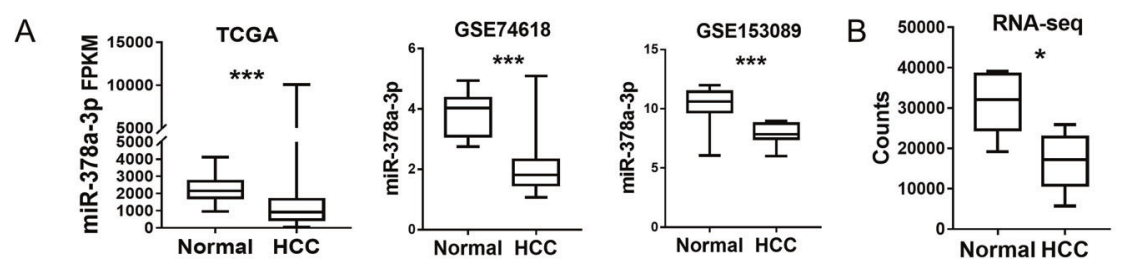

D

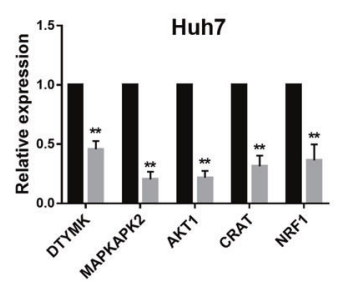

F
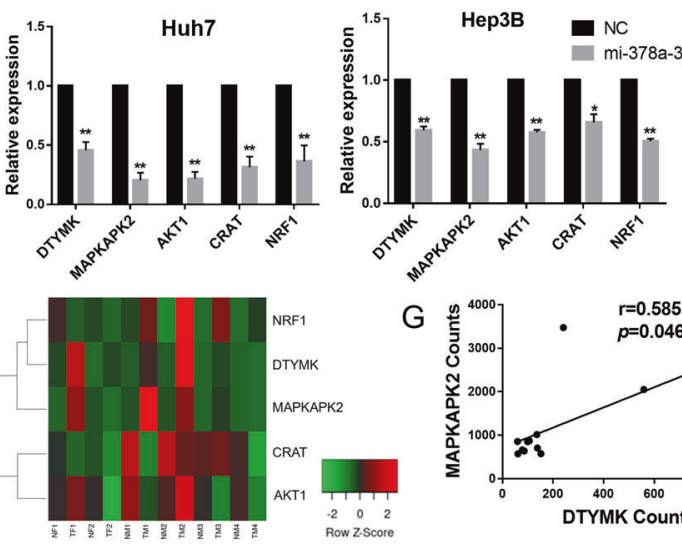

G
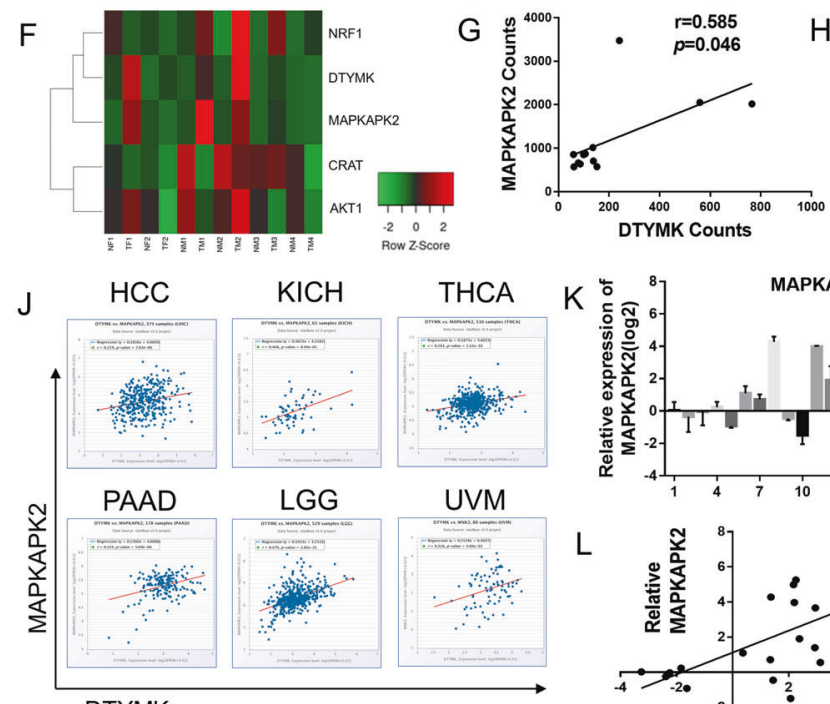

DTYMK

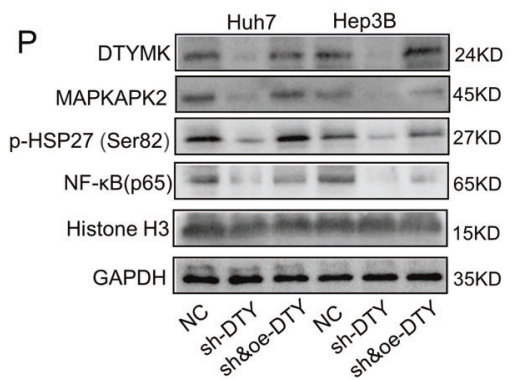

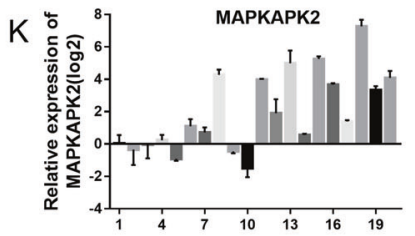

L

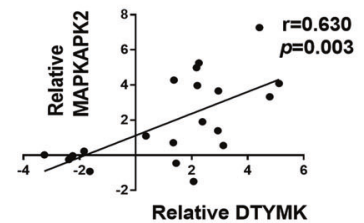

Q

DTYMK: 5' CCGUCCCCAGCAGAGUCCAGA 3' miR378a-3p: 3' GGAAGACUGAGGUUCAGGUCA MAPKAPK2: 5' GUCUCAGAUGGUGUGUCCAGC 3'

$R$

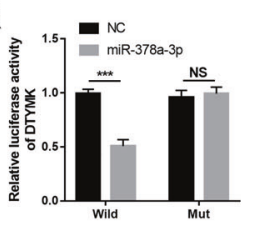
miR378a-3p: 3' G G I I 1 I I I I I ।

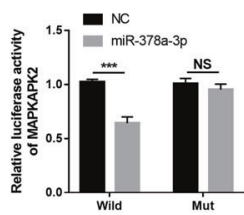

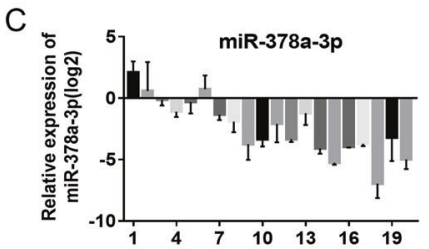
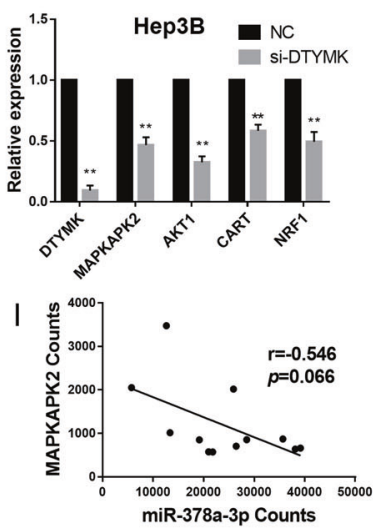
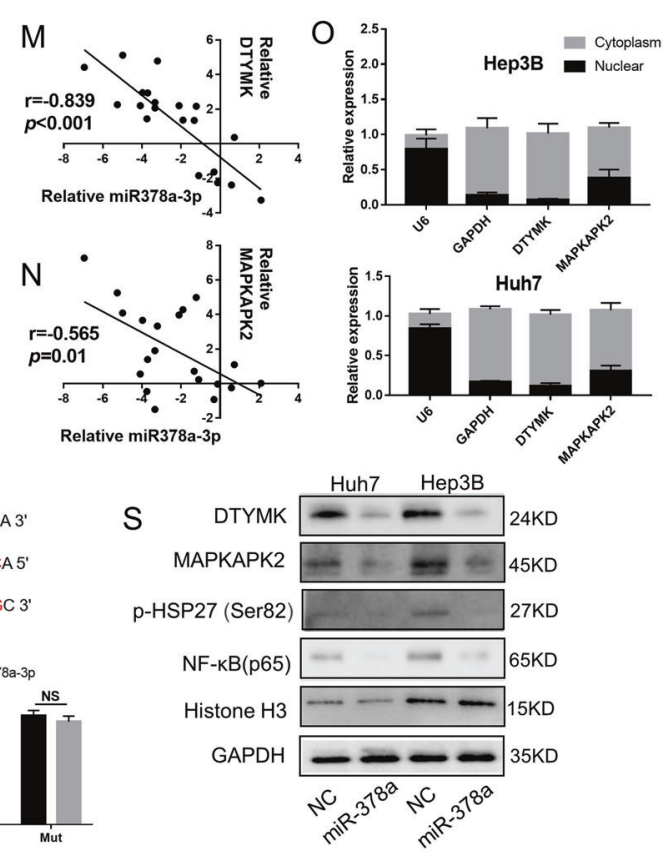

Fig. 3 DTYMK acted as ceRNA to affect the function of MAPKAPK2. A Decreased expression of miR-378a-3p in HCC from TCGA and GEO. B and C Decreased expression of miR-378a-3p in HCC from RNA-seq of 6 paired HCC tissues and qPCR of 20 paired HCC tissues. D Expression of DTYMK, MAPKAPK2, AKT1, CART, and NRF1 could be inhibited by miR-378a-3p. E Decreased expression of MAPKAPK2, AKT1, CART, and NRF1 after DTYMK knockdown. F Heatmap (RNA-seq) showed a clear correlation between DTYMK and MAPKAPK2. G Positive correlation between DTYMK and MAPKAPK2 (RNA-seq). H and I Opposite relation between miR-378a-3p, DTYMK, and MAPKAPK2 (RNA-seq). J Significant correlation between DTYMK and MAPKAPK2 in multiple cancer types. $\mathbf{K}$ and $\mathbf{L}$ Increased expression of MAPKAPK2 in 16 of 20 patients with HCC, which correlated with the expression of DTYMK. M An opposite expression trend existed between miR-378a-3p and DTYMK. N An opposite expression trend existed between miR-378a-3p and MAPKAPK2. O Subcellular locations experiments showing that DTYMK and MAPKAPK2 were enriched in the cytoplasm. P Decreased expression of MAPKAPK2 and p-hsp27, and decreased nuclear translocation of NF-KB (p65) after DTYMK knockdown. Q Potential binding sites between DTYMK, MAPKAPK2, and miR-378a-3p. R MiR-378a-3p bound and significantly inhibited the expression of DTYMK and MAPKAPK2. S MiR-378a-3p inhibited the expression of DTYMK, thus inhibiting MAPKAPK2/p-hsp27/NF-KB (p65). ${ }^{*} p<0.05 ;{ }^{* *} p<0.01 ;{ }^{* * *} p<0.001$.

by NF-kB, in the negative control group (Fig. 4F). The statistical analyze of the IHC images is showen in Fig. 4G. The enzyme-linked immunosorbent assay (ELISA) showed that the level of CCL5 in cell supernatant decreased after DTYMK-knockdown (Fig. 4H). The migration assay showed that CCL5 could stimulate the migration of human monocyte cell line THP1 and CD14 ${ }^{+}$peripheral blood monocytes (PBMC) (Fig. 4I).

\section{Increased expression of DTYMK was associated with a poor prognosis}

A total of 105 HCC paraffin-embedded tissues obtained from the Sun Yat-sen University Cancer Center were used to perform immunohistochemical assays to detect protein levels of DTYMK in HCC tissues and adjacent nontumor tissues. The results showed that the protein level of DTYMK was significantly upregulated in 
A

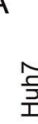

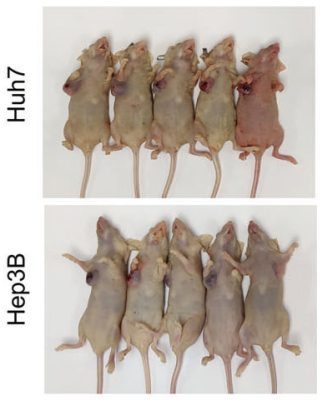

\section{B}

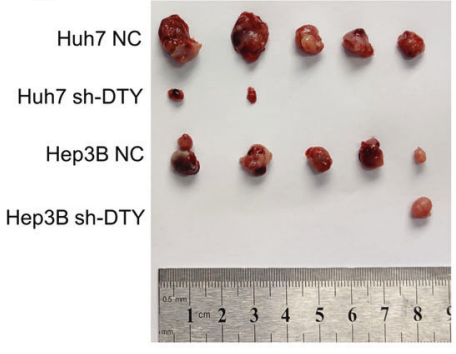

C

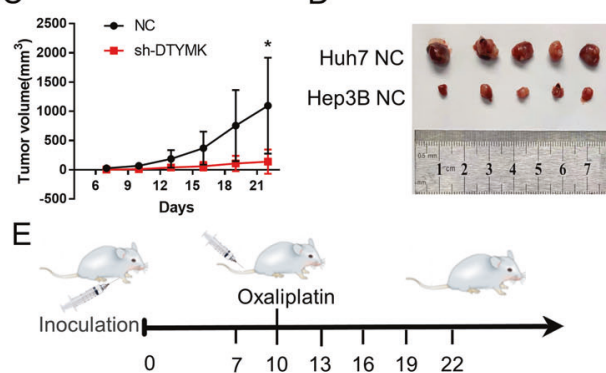

$\mathrm{F}$
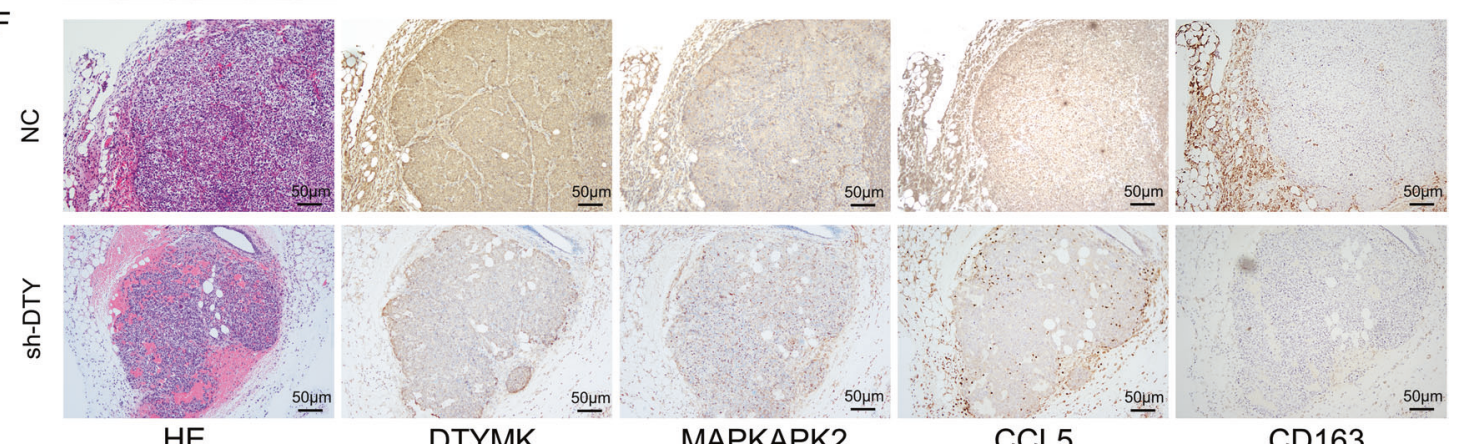

DTYMK

MAPKAPK2

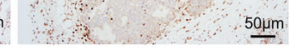

CCL5

I
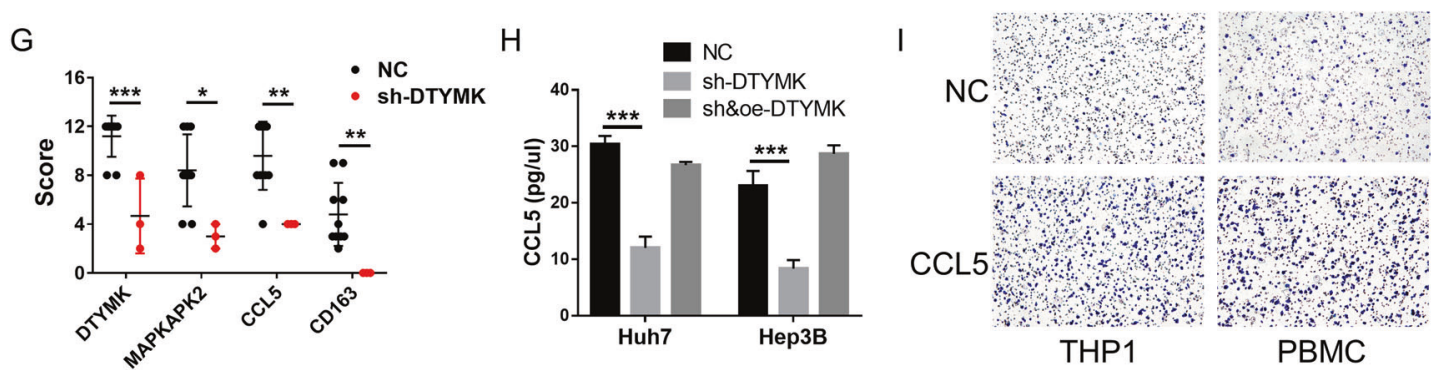

Fig. 4 Tumor formation rate and growth speed were significantly inhibited after DTYMK knockdown in vivo. A and B A stronger inhibitory effect on tumor formation rate was exhibited in transplanted carcinoma in nude mice ( $n=5$ per group). C Tumor growth speed was significantly inhibited after DTYMK knockdown $(p=0.039)$. D Tumor sizes in the negative control group decreased partially after oxaliplatin treatment, while no tumors were formatted in the sh-DTYMK group ( $n=5$ per group). E Schematic diagram of tumor xenotransplantation model. $\mathbf{F}$ The results of immunohistochemistry showed that the expression of DTYMK and MAPKAPK2 reduced in the sh-DTYMK group compared with the negative control group. Moreover, higher CCL5 expression and more CD163 ${ }^{+}$TAMs were found in the negative control group. Scale bar $=50 \mu \mathrm{m}$. G Statistical analysis of the mice IHC results. H ELISA showed an inhibited level of CCL5 after DTYMK knockdown. I CCL5 could stimulate the chemotactic migration of monocytes. NC negative control, DTY DTYMK. ${ }^{*} p<0.05 ;{ }^{* * *} p<0.01 ;{ }^{* * *} p<0.001$.

HCC tissues compared with adjacent nontumor tissues (Fig. 5A). Higher expression of DTYMK was found to correlate with poorer overall survival (OS) $(p<0.001)$ and disease-free survival (DFS) $(p=0.047)$ (Fig. 5B). Similar results were obtained from the TCGA (Fig. 5C). According to the results of the univariate and multivariate Cox regression analyses, the relative risk of DTYMK was always greater than 1 ( $p=0.009, p=0.019$, respectively), which signified that DTYMK was an independent risk factor for HCC (Table 1). The chi-square test result was shown in Supplementary Table 1. In addition, DTYMK was found to correlate with the infiltration of M2type macrophages in HCC $(r=0.262, p<0.001)$, which could promote the occurrence and development of tumors (Fig. 5D). The expression of DTYMK and the infiltration of M2-type macrophages were combined to predict the prognosis of patients with HCC more accurately, obtained using TIMER 2.0 [25] (Fig. 5E).

\section{DISCUSSION}

With the improvement in molecular biological techniques, some risk factors and molecular mechanisms of HCC have been revealed and several target drugs are being used [26-31]. However, the understanding of HCC is still uncomprehensive and the therapeutic effect is still unoptimistic $[32,33]$. The data of HCC were analyzed from the TCGA database for an in-depth understanding of the mechanism of HCC. The expression level of eight genes was significantly increased (fold change $>2$ ) in HCC, including FAM189B, DTYMK, CDC20, CDKN2C, KIFC1, PTTG1, KIF2C, and UCK2, and were associated with poor prognosis. Among these genes, DTYMK was the focus of the present study. Its role in liver cancer was unclear. Only few studies reported on the role of DTYMK, particularly in cancer occurrence and progression. The division and proliferation of tumors cannot be separated from DNA replication [34]. DTYMK has been reported to catalyze the phosphorylation of dTMP to form dTDP, which is an important material in DNA synthesis [18]. Besides, the overexpression of DTYMK has been reported to partially reverse the inhibitory effect of B029-2 on HCC cells, which is a novel p300 inhibitor [35]. In this study, the growth of Huh7 and Hep3B HCC cells and the expression of cell cycle proteins CDK2, CDK4, Cyclin A2, and Cyclin D1, which were involved in the G0/G1 and S phases, significantly reduced after DTYMK knockdown. Besides, Huh7 and Hep3B cells became more sensitive to oxaliplatin and sorafenib after DTYMK knockdown. DTYMK expression was found upregulated in 5fluorouracil-resistant derivatives, suggesting that DTYMK might be related to drug resistance in colorectal cancer cells [36]. 
A
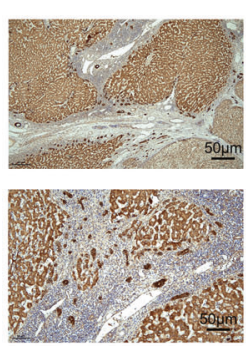

C

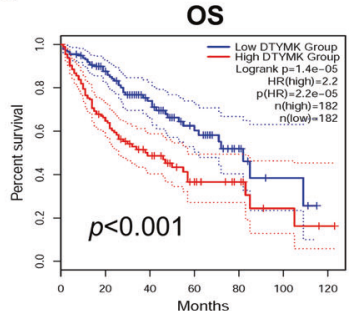

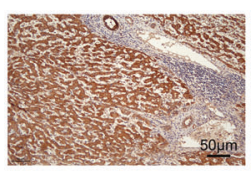

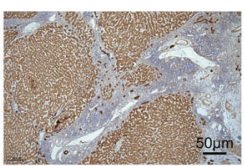

B

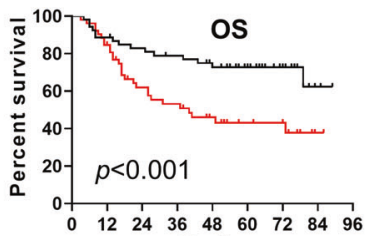

Months

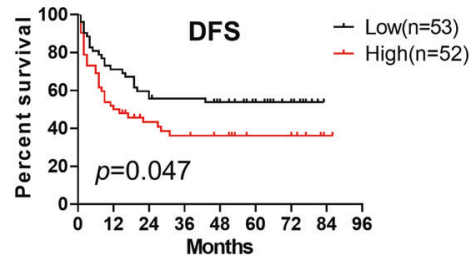

D
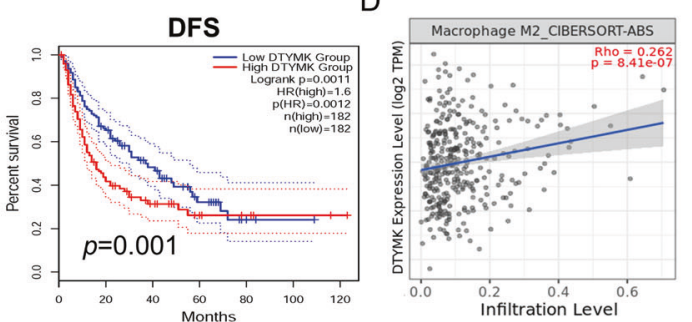

E

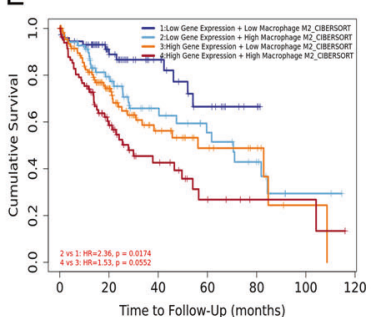

Fig. 5 DTYMK expression was significantly upregulated and associated with a poor prognosis. A Immunohistochemistry tests showing that DTYMK expression was significantly upregulated in HCC tissues. Scale bar $=50 \mu \mathrm{m}$. B Increased expression of DTYMK was associated with poor OS and DFS among 105 patients with HCC from the Sun Yat-sen University Cancer Center. C Higher expression of DTYMK was associated with poor OS and DFS in HCC from TCGA. D and E DTYMK correlated with the infiltration of M2 macrophages and predicted the prognosis of patients with HCC more accurately. OS overall survival, DFS disease-free survival.

The bioinformatics analysis showed that the expression levels of DTYMK and MAPKAPK2 highly correlated in various tumor types, and they shared the same binding site on miR-378a-3p. Then, miR$378 a-3 p$ was found to inhibit the expression of both DTYMK and MAPKAPK2. MicroRNAs (miRNAs) are a large family of posttranscriptional regulators of gene expression, with $\sim 21$ nucleotides in length and many developmental and cellular processes controlled by miRNAs in eukaryotic organisms [37]. The discovery of miRNAs opened new doors for the development of novel strategies to combat diseases. Previous studies reported that miR-378a-3p was involved in metabolism, mitochondria, and autophagy [38]. Li et al. reported that miR-378a-3p inhibited the expression of insulin-like growth factor 1 receptor in colorectal cancer cells and might play an important role as a tumor suppressor gene in the initial stage of carcinogenesis of colorectal cancer [39]. MiR-378-3p could also limit the activation of hepatic stellate cells and liver fibrosis by suppressing the expression of Gli3 [40]. No studies have reported that miR-378a-3p could inhibit DTYMK or MAPKAPK2 at present. This study was the first to verify that DTYMK and MAPKAPK2 could combine competitively with miR-378a-3p to maintain the expression of each other, and thus affect phospho-HSP27/NF-KB [41-44]. The HSP27/NF-KB axis is increasingly recognized as a crucial player in many steps of cancer initiation and progression, including immune responses, chemoresistance, and radiation-induced fibrosis [45-48]. The present study indicated that DTYMK mediated oxaliplatin resistance of HCC through the MAPKAPK2/p-HSP27/NF$K B$ pathway. Survival analysis showed that increased expression of DTYMK was associated with grade, stage, recurrence, and poor prognosis in patients with HCC. Similar to the results in vitro, the tumor formation rate and growth speed were significantly inhibited after DTYMK knockdown in nude mice. Moreover, more $\mathrm{CD}_{163^{+}} \mathrm{M}^{2}$-type TAMs were infiltrated in the negative control group than in the DTYMK knockdown group, contributing to the formation and progression of xenograft tumors $[49,50]$. Multiple studies reported that CCL5 could recruit monocytes and be regulated by NF-KB; it played essential roles in liver disease progression, especially HCC development in humans and mice [51-54]. DTYMK promoted the expression of CCL5 by affecting NF-KB, which led to increased infiltration of CD163 ${ }^{+}$M2-type TAMs in the tumor microenvironment.
In summary, DTYMK expression was upregulated and was involved in the development of HCC. Increased expression of DTYMK significantly correlated with the poor prognosis in patients with HCC. In addition, DTYMK could competitively combine with miR$378 a-3 p$ to maintain the expression of MAPKAPK2 and thus activate the phospho-HSP27/NF-KB axis, which mediated drug resistance, proliferation of tumor cells, and infiltration of $\mathrm{CD}_{163^{+}} \mathrm{M2}$-type TAMs by inducing the expression of CCL5. In previous studies, circRNAs and IncRNAs were thought to play a role through the ceRNA mechanism. The present study demonstrated a new mechanism and provided a new insight into the role of mRNA in not only encoding proteins but also participating in the ceRNA mechanism. DTYMK may be a potential biomarker and therapeutic target against HCC.

\section{MATERIALS AND METHODS}

\section{Patients and specimens}

A total of 20 pairs of HCC tissue samples and paired adjacent nontumor tissue samples were collected from the Sun Yat-sen University Cancer Center from 2018 to 2019. A total of 105 paraffin-embedded tissue sections, collected from the Sun Yat-sen University Cancer Center, were used for the immunohistochemical experiment. PBMC were collected from healthy volunteers. All experiments complied with the principles of the Declaration of Helsinki and were approved by the Research Ethics Committee of the Sun Yat-sen University Cancer Center. Informed consent was obtained from all patients to use their tissues for research purposes.

\section{Cell culture}

Hep3B HCC cell line were obtained from American Type Culture Collection (ATCC, Manassas, VA, USA); Huh7 HCC cell line was obtained from the RIKEN cell bank (Ibaraki, Japan). THP1 was provided by Tong Xiang (Sun Yat-sen University Cancer Center). THP1 and CD14 ${ }^{+}$PBMC were cultured in the Roswell Park Memorial Institute-1640 medium (Gibco, NY, USA). Huh7 and Hep3B cells were cultured in the Dulbecco's modified Eagle's medium (DMEM) (Gibco, NY, USA) supplemented with $10 \%$ fetal bovine serum (FBS) (Gibco, NY, USA) at $37^{\circ} \mathrm{C}$ in a humid atmosphere containing $5 \% \mathrm{CO}_{2}$.

\section{Transfection}

Lipofectamine 3000 (Invitrogen; Thermo Fisher Scientific, Inc.) was used to perform transfections following the manufacturer's protocol. A total of $7.5 \mu \mathrm{L}$ of Lipofectamine 3000 and final concentrations of $50 \mathrm{nM}$ of siRNA 
Table 1. Univariate and multivariate Cox regression analyses.

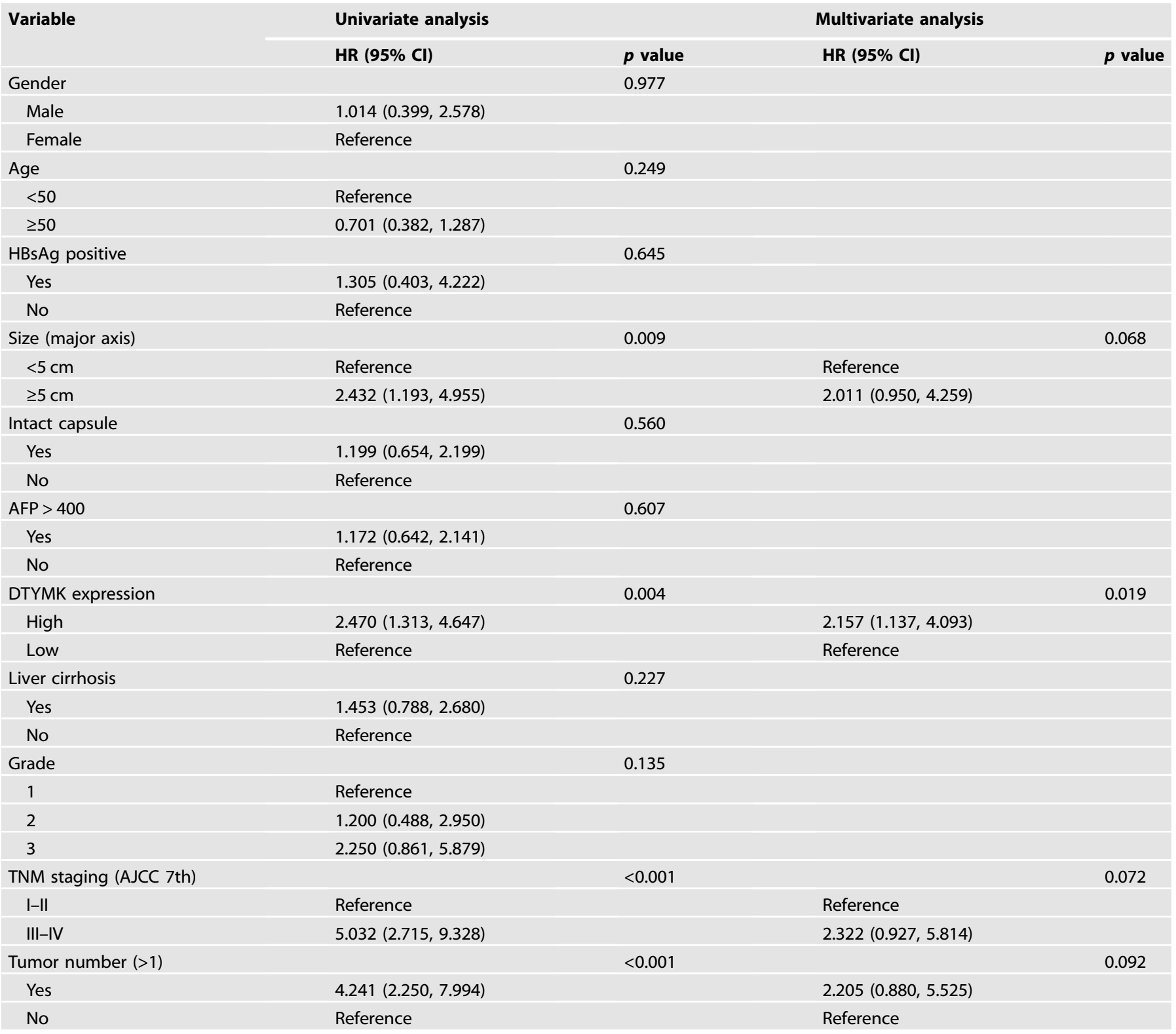

for DTYMMK (5'-CGAUGUUUAACUCGGUCAACC-3'; 5'-UUGACCGAGUUAAA CAUCGUU-3') were used for each transfection in a six-well plate with $2 \mathrm{~mL}$ of the culture medium. Knocked-down DTYMK (target sequence: GTTCCACCAGCTCATGAA) and negative control lentiviruses were obtained from Shanghai OBIO Technology (Shanghai, China). Huh7 and Hep3B cells were transfected with lentiviruses at a multiplicity of infection of 10 . After 2 weeks of $2 \mu \mathrm{g} / \mathrm{mL}$ puromycin screening, Huh7 and Hep3B cells were used for subsequent experiments. Overexpressed DTYMK and negative control plasmid with a resistance of neomycin were obtained from GeneCopoeia (Guangzhou, China).

\section{RNA isolation and real-time quantitative reverse transcriptase-polymerase chain reaction}

Total RNA was isolated using a TRIzol reagent (Invitrogen; Thermo Fisher Scientific, Inc.) following the manufacturer's protocol. The Fast All-in-One reverse transcription (RT) Kit (cat. no. ES-RT001; Shanghai Yishan Biotechnology, Co., Ltd) was used to perform RT following the manufacturer's protocol. The relative expression level of mRNAs was normalized to glyceraldehyde-3phosphate dehydrogenase (GAPDH) and calculated using the $2^{-\Delta \Delta C t}$ method. The primers used were as follows: DTYMK, forward: $5^{\prime}$-GTCCTGTTCCTCCAGT TAC-3' and reverse: 5'-AGCATCCACCATCTTCCA-3'; MAPKAPK2, forward:
5'-CGCAGTTCCACGTCAAGTC- $3^{\prime}$ and reverse: 5'-GGGCGAATTTCTCCTGGGTC3'; and GAPDH, forward: 5'-AGAAGGCTGGGGCTCATTTG-3' and reverse: 5'-AGGGGCCATCCACAGTCTTC-3'.

\section{RNA and protein isolation from the nucleus and cytoplasm}

First, up to $10^{7}$ fresh cultured cells were collected, washed once with phosphate-buffered saline (PBS), and placed on ice. A PARIS Kit (AM1921, Thermo Fisher Scientific, Inc.) was used to aspirate RNA and protein from the nucleus and cytoplasm. Then, $300 \mu \mathrm{L}$ of cell disruption buffer was added to half of the cells for total RNA. The other half of cells were resuspended in $300 \mu \mathrm{L}$ of ice-cold cell fractionation buffer and incubated on ice for $10 \mathrm{~min}$ for nuclear and cytoplasm RNA or protein. Then, the samples were centrifuged for $5 \mathrm{~min}$ at $4{ }^{\circ} \mathrm{C}$ and $500 \mathrm{~g}$, and the cytoplasmic fraction was carefully aspirated away from the nuclear pellet. The cytoplasmic lysate was cytoplasmic protein. The nuclear pellet was washed with ice-cold cell fractionation buffer. A cell disruption buffer was used to lyse the nuclear pellet. The lysate was mixed with an equal volume of $2 \times$ lysis/binding solution, and a "sample volume" of $100 \%$ ethanol was added to the mixture. The sample mixture was drawn through a filter cartridge and washed once with $700 \mu \mathrm{L}$ of wash solution 1 and twice with $500 \mu \mathrm{L}$ of wash solution 2 . In the end, RNA was eluted with $40 \mu \mathrm{L}$ of $95^{\circ} \mathrm{C}$ elution solution. 


\section{Western blot analysis}

The Western blot analysis was performed as described in a previous study [55] using anti-DTYMK antibody (15360-1-AP, Proteintech, IL, USA), antiMAPKAPK2 antibody (13949-1-AP, Proteintech, IL, USA), anti-GAPDH antibody (60004-1-lg, Proteintech, IL, USA), anti-phospho-HSP27 (Ser82) antibody (9709 T, Cell Signaling Technology, MA, USA), anti-NF-KB p65 antibody (8242 s, Cell Signaling Technology), and anti-Histone H3 antibody (Cell Signaling Technology).

\section{Cell proliferation assay}

The cell proliferation activity was measured using a Cell Counting Kit-8 (CCK-8, Dojindo Chemical Laboratory, Kumamoto, Japan). Approximately $2 \times 10^{3}$ cells in a $100 \mu \mathrm{L}$ of medium were seeded into 96-well plates after transfection. After incubation at $37^{\circ} \mathrm{C}$ for $2 \mathrm{~h}$, the absorbance at a wavelength of $450 \mathrm{~nm}$ was measured following the addition of $10 \mu \mathrm{L}$ of the CCK-8 solution.

\section{Dual-luciferase reporter assay}

The HEK293T cells were cultured in DMEM supplemented with 10\% FBS. Then, luciferase reporter vectors (GeneCopoeia, Guangzhou, China) were transfected into HEK293T cells. Next, $50 \mathrm{nM}$ of miRNA mimics or negative control mimics were transfected into HEK293T cells using Lipofectamine 3000 (Invitrogen; Thermo Fisher Scientific, Inc.). The luciferase activity was detected using the Dual-Luciferase Assay Kit (Promega, WI, USA) after $48 \mathrm{~h}$. The relative luciferase activity was normalized to Renilla luciferase activity. The bases binding to miRNA were replaced by a complementary base for mutation.

\section{Enzyme-linked immunosorbent assay}

A Human CCL5 Quantikine ELISA Kit was purchased from R\&D (DRNOOB). The ELISA measurement was carried out strictly according to the standard protocol provided by the manufacturer.

\section{Migration}

Migration experiment was carried out using the Falcon permeable support with a $3.0 \mu \mathrm{m}$ transparent PET membrane. A total of $1 \times 10^{6} \mathrm{THP} 1$ or CD14 $4^{+}$ PBMC (separation by CD14 MicroBeads, Miltenyi) resuspended in $200 \mu \mathrm{L}$ serum-free 1640 medium were seeded into the upper chamber, and a total of $500 \mu \mathrm{L}$ of 1640 medium supplemented with CCL5 $(10 \mu \mathrm{g} / \mathrm{mL})$ was added to the lower chamber. After incubation at $37^{\circ} \mathrm{C}$ with $5 \% \mathrm{CO}_{2}$ for $4 \mathrm{~h}$, the cells that passed through the membrane were fixed with $4 \%$ formaldehyde for $30 \mathrm{~min}$ and washed with PBS two times, and then stained with $0.1 \%$ crystal violet for $20 \mathrm{~min}$ and washed with water.

\section{Animal experiments}

Five-week-old female BALB/c-nu/nu mice, purchased from Guangdong Medical Laboratory Center (China), were randomly divided into four groups ( $n=5$ in each group). A total of $2 \times 10^{6}$ DTYMK-knockdown Hep3B or Huh7 cells in $0.15 \mathrm{~mL}$ of PBS were subcutaneously injected into the right armpit region of the mice, and negative control cells were subcutaneously injected into the other side. The tumor size was measured every 3 days from the 7 th day after injection. Oxaliplatin $(5 \mathrm{mg} / \mathrm{kg})$ was injected through the tail vein every 3 days from the 10th day after injection. After 28 days of injection, the mice were sacrificed, and tumors were isolated and measured. Animal experiments were approved by the animal ethics committee of the Sun Yat-sen University Cancer Center.

\section{Statistical analysis}

Statistical analyses were performed using SPSS 20.0 (IBM Corp). Graphs were generated using GraphPad Prism 7 (GraphPad Software, Inc). Pan-cancer view and association analysis between DTYMK expression, stages, and grades were performed by UALCAN [56]. Survival analysis of data from TCGA was performed by GEPIA [57]. The unpaired-sample Student $t$ test was used to evaluate the differences between the two groups of independent samples. Data were presented as mean \pm standard deviation. All the results were repeated more than three times. A $p$ value $<0.05$ from a two-tailed test was considered to indicate a statistically significant difference. Statistical significance was indicated as ${ }^{*} p<0.05,{ }^{* *} p<0.01$, and ${ }^{* * *} p<0.001$.

\section{DATA AVAILABILITY}

All data generated during this study and datasets used and/or analyzed during the current study are available from the corresponding author on reasonable request.

\section{REFERENCES}

1. Siegel RL, Miller KD, Fuchs HE, Jemal A. Cancer Statistics, 2021. CA Cancer J Clin. 2021;71:7-33.

2. El-Serag HB. Hepatocellular carcinoma. N Engl J Med. 2011;365:1118-27.

3. Fujiwara N, Friedman SL, Goossens N, Hoshida Y. Risk factors and prevention of hepatocellular carcinoma in the era of precision medicine. J Hepatol. 2018;68:526-49.

4. Wang S, Feng Y, Swinnen J, Oyen R, Li Y, Ni Y. Incidence and prognosis of liver metastasis at diagnosis: a pan-cancer population-based study. Am J Cancer Res. 2020;10:1477-517.

5. Costentin CE, Bababekov YJ, Zhu AX, Yeh H. Is It Time to Reconsider the Milan Criteria for Selecting Patients With Hepatocellular Carcinoma for Deceased-Donor Liver Transplantation? Hepatology. 2019;69:1324-36.

6. Finn RS, Zhu AX, Farah W, Almasri J, Zaiem F, Prokop $U$, et al. Therapies for advanced stage hepatocellular carcinoma with macrovascular invasion or metastatic disease: A systematic review and meta-analysis. Hepatology. 2018;67:422-35.

7. Forner A, Reig M, Bruix J. Hepatocellular carcinoma. Lancet. 2018;391:1301-14.

8. Villanueva A. Hepatocellular Carcinoma. N Engl J Med. 2019;380:1450-62.

9. Zhou B, Yan Z, Liu R, Shi P, Qian S, Qu X, et al. Prospective Study of Transcatheter Arterial Chemoembolization (TACE) with Ginsenoside Rg3 versus TACE Alone for the Treatment of Patients with Advanced Hepatocellular Carcinoma. Radiology. 2016;280:630-9.

10. Lyu N, Kong $Y, M u ~ L$, Lin $Y$, Li J, Liu Y, et al. Hepatic arterial infusion of oxaliplatin plus fluorouracil/leucovorin vs. sorafenib for advanced hepatocellular carcinoma. J Hepatol. 2018;69:60-9.

11. Goyal L, Zheng H, Abrams TA, Miksad R, Bullock AJ, Allen JN, et al. A Phase II and Biomarker Study of Sorafenib Combined with Modified FOLFOX in Patients with Advanced Hepatocellular Carcinoma. Clin Cancer Res. 2019;25:80-9.

12. Liao X, Bu Y, Jiang S, Chang F, Jia F, Xiao X, et al. CCN2-MAPK-Id-1 loop feedback amplification is involved in maintaining stemness in oxaliplatin-resistant hepatocellular carcinoma. Hepatol Int. 2019;13:440-53.

13. Ding ZB, Hui B, Shi YH, Zhou J, Peng YF, Gu CY, et al. Autophagy activation in hepatocellular carcinoma contributes to the tolerance of oxaliplatin via reactive oxygen species modulation. Clin Cancer Res. 2011;17:6229-38.

14. Kohnken R, Kodigepalli KM, Wu L. Regulation of deoxynucleotide metabolism in cancer: novel mechanisms and therapeutic implications. Mol Cancer. 2015;14:176.

15. Van Triest B, Pinedo HM, Giaccone G, Peters GJ. Downstream molecular determinants of response to 5 -fluorouracil and antifolate thymidylate synthase inhibitors. Ann Oncol. 2000;11:385-91.

16. Reichard P. Interactions between deoxyribonucleotide and DNA synthesis. Annu Rev Biochem. 1988;57:349-74.

17. Arner ES, Eriksson S. Mammalian deoxyribonucleoside kinases. Pharm Ther. 1995;67:155-86

18. Liu Y, Marks K, Cowley GS, Carretero J, Liu Q, Nieland TJ, et al. Metabolic and functional genomic studies identify deoxythymidylate kinase as a target in LKB1mutant lung cancer. Cancer Disco. 2013;3:870-9.

19. Oliver FJ, de la Rubia G, Rolli V, Ruiz-Ruiz MC, de Murcia G, Murcia JM. Importance of poly(ADP-ribose) polymerase and its cleavage in apoptosis. Lesson from an uncleavable mutant. J Biol Chem. 1998;273:33533-9.

20. Korsmeyer SJ, Shutter JR, Veis DJ, Merry DE, Oltvai ZN. Bcl-2/Bax: a rheostat that regulates an anti-oxidant pathway and cell death. Semin Cancer Biol. 1993;4:327-32.

21. Li JH, Liu S, Zhou H, Qu LH, Yang JH. starBase v2.0: decoding miRNA-ceRNA, miRNA-ncRNA and protein-RNA interaction networks from large-scale CLIP-Seq data. Nucleic Acids Res. 2014;42:D92-97. Database issue

22. Yang Q, Zhao S, Shi Z, Cao L, Liu J, Pan T, et al. Chemotherapy-elicited exosomal miR-378a-3p and miR-378d promote breast cancer stemness and chemoresistance via the activation of EZH2/STAT3 signaling. J Exp Clin Cancer Res. 2021;40:120.

23. Martinez-Quetglas I, Pinyol R, Dauch D, Torrecilla S, Tovar V, Moeini A, et al. IGF2 Is Up-regulated by Epigenetic Mechanisms in Hepatocellular Carcinomas and Is an Actionable Oncogene Product in Experimental Models. Gastroenterology. 2016;151:1192-205.

24. Honda $S$, Chatterjee $A$, Leichter AL, Miyagi $H$, Minato $M$, Fujiyoshi $S$, et al. $A$ MicroRNA Cluster in the DLK1-DIO3 Imprinted Region on Chromosome 14q32.2 Is Dysregulated in Metastatic Hepatoblastomas. Front Oncol. 2020;10:513601.

25. Li T, Fu J, Zeng Z, Cohen D, Li J, Chen Q, et al. TIMER2.0 for analysis of tumorinfiltrating immune cells. Nucleic Acids Res. 2020;48:W509-W514.

26. Rimassa L, Assenat E, Peck-Radosavljevic M, Pracht M, Zagonel V, Mathurin P, et al. Tivantinib for second-line treatment of MET-high, advanced hepatocellular carcinoma (METIV-HCC): a final analysis of a phase 3, randomised, placebocontrolled study. Lancet Oncol. 2018;19:682-93.

27. Bruix J, Qin S, Merle P, Granito A, Huang YH, Bodoky G, et al. Regorafenib for patients with hepatocellular carcinoma who progressed on sorafenib treatment (RESORCE): a randomised, double-blind, placebo-controlled, phase 3 trial. Lancet. 2017;389:56-66. 
28. Simon TG, Ma Y, Ludvigsson JF, Chong DQ, Giovannucci EL, Fuchs CS, et al. Association Between Aspirin Use and Risk of Hepatocellular Carcinoma. JAMA Oncol. 2018;4:1683-90.

29. Yang HI, Yuen MF, Chan HL, Han KH, Chen PJ, Kim DY, et al. Risk estimation for hepatocellular carcinoma in chronic hepatitis $B(\mathrm{REACH}-\mathrm{B})$ : development and validation of a predictive score. Lancet Oncol. 2011;12:568-74.

30. Yang W, Ma Y, Liu Y, Smith-Warner SA, Simon TG, Chong DQ, et al. Association of Intake of Whole Grains and Dietary Fiber With Risk of Hepatocellular Carcinoma in US Adults. JAMA Oncol. 2019;5:879-86.

31. Jarvis H, Craig D, Barker R, Spiers G, Stow D, Anstee QM, et al. Metabolic risk factors and incident advanced liver disease in non-alcoholic fatty liver disease (NAFLD): A systematic review and meta-analysis of population-based observational studies. PLoS Med. 2020;17:e1003100.

32. Xiang DM, Sun W, Zhou T, Zhang C, Cheng Z, Li SC, et al. Oncofetal HLF transactivates c-Jun to promote hepatocellular carcinoma development and sorafenib resistance. Gut. 2019;68:1858-71.

33. Huo YR, Eslick GD. Transcatheter Arterial Chemoembolization Plus Radiotherapy Compared With Chemoembolization Alone for Hepatocellular Carcinoma: A Systematic Review and Meta-analysis. JAMA Oncol. 2015;1:756-65.

34. Macheret $M$, Halazonetis TD. DNA replication stress as a hallmark of cancer. Annu Rev Pathol. 2015;10:425-48.

35. Cai LY, Chen SJ, Xiao SH, Sun QJ, Ding CH, Zheng BN, et al. Targeting p300/CBP attenuates hepatocellular carcinoma progression through epigenetic regulation of metabolism. Cancer Res. 2021;81:860-72. https://doi.org/10.1158/0008-5472. CAN-20-1323.

36. de Angelis PM, Fjell B, Kravik KL, Haug T, Tunheim SH, Reichelt W, et al. Molecular characterizations of derivatives of HCT116 colorectal cancer cells that are resistant to the chemotherapeutic agent 5-fluorouracil. Int J Oncol. 2004;24:1279-88.

37. Krol J, Loedige I, Filipowicz W. The widespread regulation of microRNA biogenesis, function and decay. Nat Rev Genet. 2010;11:597-610.

38. Machado IF, Teodoro JS, Palmeira CM, Rolo AP. miR-378a: a new emerging microRNA in metabolism. Cell Mol Life Sci. 2020;77:1947-58.

39. Li H, Dai S, Zhen T, Shi $H$, Zhang F, Yang $Y$, et al. Clinical and biological significance of miR-378a-3p and miR-378a-5p in colorectal cancer. Eur J Cancer. 2014;50:1207-21.

40. Hyun J, Wang S, Kim J, Rao KM, Park SY, Chung I, et al. MicroRNA-378 limits activation of hepatic stellate cells and liver fibrosis by suppressing Gli3 expression. Nat Commun. 2016;7:10993.

41. Wu J, Liu T, Rios Z, Mei Q, Lin X, Cao S. Heat Shock Proteins and Cancer. Trends Pharm Sci. 2017;38:226-56.

42. Zheng G, Zhang Z, Liu H, Xiong Y, Luo L, Jia X, et al. HSP27-Mediated Extracellular and Intracellular Signaling Pathways Synergistically Confer Chemoresistance in Squamous Cell Carcinoma of Tongue. Clin Cancer Res. 2018;24:1163-75.

43. Calderwood SK, Gong J. Heat Shock Proteins Promote Cancer: It's a Protection Racket. Trends Biochem Sci. 2016;41:311-23.

44. Henriques A, Koliaraki V, Kollias G. Mesenchymal MAPKAPK2/HSP27 drives intestinal carcinogenesis. Proc Natl Acad Sci USA. 2018;115:E5546-E5555.

45. Crane JD, Ogborn DI, Cupido C, Melov S, Hubbard A, Bourgeois JM, et al. Massage therapy attenuates inflammatory signaling after exercise-induced muscle damage. Sci Transl Med. 2012;4:119ra113.

46. Kim JY, Jeon S, Yoo YJ, Jin H, Won HY, Yoon K, et al. The Hsp27-Mediated IkBalpha-NFkappaB Signaling Axis Promotes Radiation-Induced Lung Fibrosis. Clin Cancer Res. 2019;25:5364-75.

47. Hoesel B, Schmid JA. The complexity of NF-kappaB signaling in inflammation and cancer. Mol Cancer. 2013;12:86.

48. DiDonato JA, Mercurio F, Karin M. NF-kappaB and the link between inflammation and cancer. Immunol Rev. 2012;246:379-400.

49. Zizzo G, Hilliard BA, Monestier M, Cohen PL. Efficient clearance of early apoptotic cells by human macrophages requires $\mathrm{M} 2 \mathrm{c}$ polarization and MerTK induction. $J$ Immunol. 2012;189:3508-20.

50. Pollard JW. Tumour-educated macrophages promote tumour progression and metastasis. Nat Rev Cancer. 2004:4:71-8.

51. Chaturvedi P, Gilkes DM, Takano N, Semenza GL. Hypoxia-inducible factordependent signaling between triple-negative breast cancer cells and mesenchymal stem cells promotes macrophage recruitment. Proc Natl Acad Sci USA. 2014;111:E2120-2129.

52. Yeo H, Lee YH, Koh D, Lim Y, Shin SY. Chrysin Inhibits NF-kappaB-Dependent CCL5 Transcription by Targeting IkappaB Kinase in the Atopic Dermatitis-Like Inflammatory Microenvironment. Int J Mol Sci. 2020;21:7348.

53. Fitzgerald KA, McWhirter SM, Faia KL, Rowe DC, Latz E, Golenbock DT, et al. IKKepsilon and TBK1 are essential components of the IRF3 signaling pathway. Nat Immunol. 2003;4:491-6.
54. Mohs A, Kuttkat N, Reissing J, Zimmermann HW, Sonntag R, Proudfoot A, et al. Functional role of CCL5/RANTES for HCC progression during chronic liver disease. J Hepatol. 2017;66:743-53.

55. Kim B. Western Blot Techniques. Methods Mol Biol. 2017;1606:133-9.

56. Chandrashekar DS, Bashel B, Balasubramanya SAH, Creighton CJ, PonceRodriguez I, Chakravarthi B, et al. UALCAN: A Portal for Facilitating Tumor Subgroup Gene Expression and Survival Analyses. Neoplasia. 2017;19:649-58.

57. Tang Z, Li C, Kang B, Gao G, Li C, Zhang Z. GEPIA: a web server for cancer and normal gene expression profiling and interactive analyses. Nucleic Acids Res. 2017;45:W98-W102.

\section{AUTHOR CONTRIBUTIONS}

FS participated in the research design and carried out the study. FS and TG participated in manuscript preparation. $Y L, T G, H C$, and $Y C$ participated in the experimental study. JX, RP, TX, and DW participated in research design and reviewed the paper. JZ and $\mathrm{YH}$ provided RNA-sequencing data. $\mathrm{YT}, \mathrm{YH}, \mathrm{JY}, \mathrm{HL}, \mathrm{CY}, \mathrm{QW}, \mathrm{YL}$, and $\mathrm{JH}$ provided partial antibodies, reagents, and tissue sample support for this experiment.

\section{FUNDING}

This study was supported by the National Key R\&D Program of China (grant number 2018YFC1313400 to JCX), the National Natural Science Foundation of China (grant number 81773110 to JCX), the Science and Technology Planning Project of Guangdong Province, China (grant number $2017 \mathrm{~B} 020227003$ to JCX), the Science and Technology Planning Project of Guangzhou, Guangdong Province, China (grant number 201704020215 to JCX), and the District High Tech R\&D Funds of Guangzhou, Guangdong Province, China (grant number 2017-L179 to JCX).

\section{COMPETING INTERESTS}

The authors declare no competing interests.

\section{ETHICS APPROVAL AND CONSENT TO PARTICIPATE}

Ethics approval (approval no. 21020S) was obtained from the Research Ethics Committee of the Sun Yat-sen University Cancer Center. Informed consent was obtained from all patients.

\section{ADDITIONAL INFORMATION}

Supplementary information The online version contains supplementary material available at https://doi.org/10.1038/s41419-021-04375-3.

Correspondence and requests for materials should be addressed to Ruiqing Peng or Jianchuan Xia.

Reprints and permission information is available at http://www.nature.com/ reprints

Publisher's note Springer Nature remains neutral with regard to jurisdictional claims in published maps and institutional affiliations.

(i) Open Access This article is licensed under a Creative Commons Attribution 4.0 International License, which permits use, sharing, adaptation, distribution and reproduction in any medium or format, as long as you give appropriate credit to the original author(s) and the source, provide a link to the Creative Commons license, and indicate if changes were made. The images or other third party material in this article are included in the article's Creative Commons license, unless indicated otherwise in a credit line to the material. If material is not included in the article's Creative Commons license and your intended use is not permitted by statutory regulation or exceeds the permitted use, you will need to obtain permission directly from the copyright holder. To view a copy of this license, visit http://creativecommons. org/licenses/by/4.0/.

(c) The Author(s) 2021 